\title{
Daylight saving for the brain
}

\author{
James Cully \\ School of Applied Psychology, UCC
}

Learning never exhausts the mind. Leonardo Da Vinci

\section{Learning throughout life}

Our lives are comprised of our doings. We love, we lose, we learn and we forget, and throughout we are dependent on our brains to maintain a level of cognitive functioning (such as short and long term memory, attention to specific sensations and speaking and understanding language) that allows us to live our lives in the manner we want. If we lose this level of functioning we are bereft and vulnerable. Growing old is something to which every healthy human aspires. More and more people are reaching old-age; the ageing of the world's population is considered mankind's greatest achievement of the 20th century. It has also become one of the main health challenges in modern medicine; diseases associated with ageing, such as Alzheimer's disease (AD), have increased drastically in rate. One of the main consequences of developing $\mathrm{AD}$ is that your brain's functional capacity decreases. You forget more and more as it progresses, losing the ability to think, to plan, to function in a manner commensurate with your life to that point. You begin to become helpless. But research has found that we are not completely helpless, not always at the mercy of the absurd, relative to developing $\mathrm{AD}$. How we live and what we do has an impact on the risk of developing AD.

\section{Tom and Jerry}

In a relatively large village in Cork, Ireland, two men in their early eighties passed away within a year of each other. Both men, Tom and Jerry, had spent all their lives in this village. Like the majority of the local community of their generation, once it was possible to leave school they went to work in the nearby factory. Tom and Jerry were friendly but rarely socialised much outside of work other than the odd staff night out. Tom was very active in his spare time, always engaged in some community building project or chairing a book club. He sang rather badly, but still took part in the amateur production of a number of plays during his 50's, organised by the local community centre. Two years ago he bought an iPad, feeling it necessary to remain in contact with his grandchildren. He had a smartphone for a year before this and so it proved relatively straightforward for him to master. Tom frequented a local cafe daily for breakfast, where he would read the Irish Times before rushing off to meet a friend for a walk, or attend a meeting in the local 
Men's Shed group, a social activity group for men aged 50 or older. Jerry was always more reserved in his leisure activities. When he was employed he would normally head for a few drinks on a Thursday night, every other night he'd unwind at home in front of the television. He was strongly opinionated regarding the "new" technology that had become ubiquitous in society, insisting he was too old to bother learning something so alien to him. Jerry's trips to the pub began to lose their appeal over the last decade of his life; it became very difficult for him to keep track of the conversations, to remember the names of the people he spent many a long night conversing with. The world he once knew became a puzzle for Jerry and he was moved to a residential care unit on the outskirts of the village where he spent the last 4 years of his life.

When both men died they underwent autopsies as part of a study on ageing. Jerry was suffering $\mathrm{AD}$ at the time of his death, diagnosed as being in the middle-stage of the disease. His autopsy highlighted this; the neuropathological damage was consistent with his level of cognitive functioning. Tom was deemed cognitively healthy right up until the time of his death, performing slightly above the average level of performance for someone of his age. Yet, when an autopsy was performed it was found that the amount of neuropathological damage his brain had suffered was similar to that of Jerry's. How can this be explained?

\section{Cognitive reserve}

The cognitive reserve hypothesis postulates that individuals differ in the level of neuropathology they can endure before they show symptoms leading to a clinical diagnosis of AD. The hypothesis developed as a result of a series of startling discoveries that were made at the end of the 1980's. Studies such as that by Katzman and colleagues focused on autopsies of the brains of recently deceased older adults whose cognitive functioning had been measured during the final years of their lives. Certain brains showed levels of pathology that should have severely impacted participants' level of cognitive functioning and resulted in a clinical diagnosis of $\mathrm{AD}$. Instead these individuals exhibited a level cognitive functioning equal to that of people of the same age who showed no signs of $A D$ pathology upon their death. The authors termed this a "reserve", suggesting that it was called upon by the brain to compensate for damage.

There are two main models of cognitive reserve: the hardware model and the software model. The hardware model of cognitive reserve is a purely quantitative model of that suggests that people with bigger, heavier, brains are able to withstand higher levels of damage before a decrease in cognitive functioning is observed. This approach, known as Brain Reserve, assumes that there is a quantitative threshold, a limit of damage that can be incurred, before cognitive functioning is impacted. The software model of cognitive reserve is inherently different as it focuses on what is left of the brain and how it adapts to pathological changes to certain parts of the brain. The two main facets of the software model of cognitive reserve are Neural Reserve and Neural Compensation. Neural Reserve 
explains the difference in levels of efficiency in brains, such that there is individual variation in levels of neuronal activation necessary for the completion of tasks. Higher Neural Reserve results in a higher capacity for activations for difficult tasks. Neural Compensation is the means by which the brain can complete tasks by recruiting different parts of the brain to complete a task that was initially done by a now-damaged brain area.

\section{Improving cognitive reserve}

Having a higher level of cognitive functioning is considered a proxy of a higher level of cognitive reserve. Cognitive functioning is measured by a variety of different tasks, and so improvement on these tasks is equated with a higher level of cognitive functioning. Research has identified a number of different variables that affect a person's level of cognitive functioning including education, occupation and the level of cognitive activities they can be engaged in as part of their lifestyle. There is a substantial body of cross-sectional evidence that highlights this strong association between having a high level of cognitive functioning and a decreased risk of developing $\mathrm{AD}$. My research focuses on cognitive activities, and how they contribute to cognitive reserve. Cognitive activities as part of your lifestyle, such as reading, writing, doing jigsaws, playing crosswords, creating art, using an iPad and playing music are all examples of activities we engage in that may improve or sustain your level of cognitive functioning. I say may because as of yet there is not enough empirical evidence to make a case for or against a cognitively engaged lifestyle as a means of maintaining your cognitive functioning. There is a strong association between the two, but causality is an area of contention. One of the main aims of my research is to develop a programme of cognitive activities that target specific cognitive functions such as verbal fluency, short-term and long-term memory and then measure whether or not a person's cognitive functioning is improved by these activities.

\section{Controlling the noise}

The overarching theme of the research it to attempt to improve cognitive functioning through everyday activities that engage the mind. Recent research by Park et al. has found that taking up a novel activity that requires a detailed level of learning, such as digital photography or an iPad, can have a positive influence on our episodic memory. Episodic memory is a form of long term-memory that is autobiographical, concerned with personal experiences of the past, in essence our personal history. To carry out experiments that test the impact of certain activities on a person's cognitive functioning in a scientific manner requires that all factors which may influence a person's level of cognitive functioning be controlled for. For instance two elderly people given an iPad and tested on cognitive functioning at the outset and two months after may show very different changes in their level of cognitive functioning between the first and second test. There are many other variables that must be considered before changes in functioning are compared for both 
people. One person may be suffering from depression and find it difficult to motivate themselves to practice this new task. Or they may have a low level of education and feel intimidated by the complicated nature of the task. Before the test of efficacy of everyday tasks it is imperative that all factors that can impact such improvements are known and measured during the experiments.

\section{Study 1.1: The impact of perceptions of ageing on lifestyle}

The first study in my $\mathrm{PhD}$ began in March. It focused on investigating the possible association between how middle/old aged people feel about the process of growing old and their cognitive lifestyle. This age-group was selected as it is an age period of transition, when we are nearing the end of our careers, nearing the beginning of old-age. How we feel about ageing, such as whether we think that we grow wiser with age or not, has been found to have an impact on many facets of a person's life. Research has found that people with a more negative perception of ageing in young and middle adulthood are more likely to suffer a decline in memory performance over the lifetime than those with a positive perception. The study comprised of a questionnaire that measured a number of different variables such as a person's perception of ageing, their level of cognitive activity and how serious or frivolous they believe certain activities, such as reading novels, can be. Questions pertaining to a person's primary occupation over the lifetime, what age they were, whether they are in receipt of certain medication, how long they spent in formal education were asked to compare these demographics. A scale that measured depression was also included in the questionnaire as previous research has found that depression can have an effect on how much time a person spends engaged in cognitive activities.

The results that emerged from Study 1.1 evidenced a strong association between a person's perception of ageing and their level of cognitive activity. People with a more positive perception of ageing were found to have a higher level of cognitive activity compared to those with a negative perception of ageing, when controlling for all other factors. This is the first time this association has been studied and so is an interesting finding. The next step in the research is to ascertain whether or not improving a person's perception of ageing in an experimental setting will mean that they spend more time engaged in a cognitively engaging task.

\section{A battery of cognitive programmes}

Once these experiments are complete I will begin the intervention programmes. The results of study 1.1 and 1.2 will be used to structure the interventions; because perception of ageing has been found to correlate with cognitive activity it will be controlled for in the interventions. Study 1.2 is an extension of Study 1.1 and will investigate if the results of Study 1.1 are replicated in an experimental setting. The interventions which I aim to run 
are eclectic in terms of the type of activities. One planned intervention is a paired-reading style study where older adults would help children to read in a school setting, while another is to have participants engage in an intensive iPad learning course. Recent studies have shown improvements in skills specifically targeted by cognitive training. Unfortunately most of these studies have suffered from a lack of transference of the skills learned from these tests to everyday cognitive functioning. The aim of my research is to provide empirical evidence of cognitively engaging activities that improve general cognitive functioning. Recent research has found that novel and challenging cognitive activities have the potential to improve cognitive functioning not specifically targeted in older adults, and so I wish to emulate these studies while also accounting for a person's perception of ageing throughout the study. Achieving such results has the potential to ameliorate the current knowledge surrounding the association between $\mathrm{AD}$ and our everyday lives, thus moving us a step closer to understanding how best this disease can be delayed, or even prevented. Providing solid empirical data that shows that a varied and engaging cognitive lifestyle throughout middle and old age can sustain or even improve cognitive functioning may serve as an impetus for people to engage in such activities, a worthwhile pursuit.

Thanks to my supervisor, Dr. Mike Murphy, and to all the participants who have contributed to this research. 